\title{
Spatiotemporal changes in diffusivity and anisotropy in fetal brain tractography
}

\author{
Fedel Machado-Rivas ${ }^{1}$ ( ) | Onur Afacan ${ }^{1}$ (ㅇ | Shadab Khan ${ }^{1}$ | Bahram Marami $^{1}$ | \\ Clemente Velasco-Annis $^{1}$ | Hart Lidov ${ }^{2}$ | Simon K. Warfield ${ }^{1}$ (i) | Ali Gholipour ${ }^{1}$ () | \\ Camilo Jaimes ${ }^{1}$ (
}

${ }^{1}$ Computational Radiology Laboratory (CRL), Department of Radiology, Boston Children's Hospital, and Harvard Medical School, Boston, Massachusetts, USA

${ }^{2}$ Department of Pathology, Boston Children's Hospital, and Harvard Medical School, Boston, Massachusetts, USA

\section{Correspondence}

Camilo Jaimes, Computational Radiology Laboratory (CRL), Department of Radiology, Boston Children's Hospital, and Harvard Medical School, Boston, Massachusetts, USA. Email: camilo.jaimescobos@childrens. harvard.edu

\section{Funding information}

Fetal Health Foundation; McKnight Foundation; National Institute of Biomedical Imaging and Bioengineering, Grant/Award Numbers: R01EB013248, R01EB018988, R01EB031849; National Institute of Dental and Craniofacial Research, Grant/Award Number: R03DE022109; National Institute of Neurological Disorders and Stroke, Grant/ Award Number: R01NS106030; National Institutes of Health, Grant/Award Number: S10OD0250111; Schlaeger Fellowship for Neuroscience Research

\begin{abstract}
Population averaged diffusion atlases can be utilized to characterize complex microstructural changes with less bias than data from individual subjects. In this study, a fetal diffusion tensor imaging (DTI) atlas was used to investigate tract-based changes in anisotropy and diffusivity in vivo from 23 to 38 weeks of gestational age (GA). Healthy pregnant volunteers with typically developing fetuses were imaged at $3 \mathrm{~T}$. Acquisition included structural images processed with a super-resolution algorithm and DTI images processed with a motion-tracked slice-to-volume registration algorithm. The DTI from individual subjects were used to generate 16 templates, each specific to a week of GA; this was accomplished by means of a tensor-to-tensor diffeomorphic deformable registration method integrated with kernel regression in age. Deterministic tractography was performed to outline the forceps major, forceps minor, bilateral corticospinal tracts (CST), bilateral inferior fronto-occipital fasciculus (IFOF), bilateral inferior longitudinal fasciculus (ILF), and bilateral uncinate fasciculus (UF). The mean fractional anisotropy (FA) and mean diffusivity (MD) was recorded for all tracts. For a subset of tracts (forceps major, CST, and IFOF) we manually divided the tractograms into anatomy conforming segments to evaluate within-tract changes. We found tract-specific, nonlinear, age related changes in FA and MD. Early in gestation, these trends appear to be dominated by cytoarchitectonic changes in the transient white matter fetal zones while later in gestation, trends conforming to the progression of myelination were observed. We also observed significant (local) heterogeneity in within-tract developmental trajectories for the CST, IFOF, and forceps major.
\end{abstract}

\section{KEYWORDS}

anisotropy, atlas, diffusion, fetal, myelination, tractography 


\section{1 | INTRODUCTION}

Development of the fetal brain in the second and third trimesters is remarkably complex. In addition to rapid growth, a series of cellular changes take place, including neuronal proliferation and migration, axonal growth and pruning, dendritic sprouting, synapse formation, and myelination (Andescavage et al., 2017; Brody, Kinney, Kloman, \& Gilles, 1987; Bystron, Blakemore, \& Rakic, 2008). These processes are frequently analyzed in the context of findings on structural in vivo and ex vivo fetal MRI and include the progressive decrease in volume of the proliferative compartment (ventricular and subventricular zone) after the 25th week of gestational age (GA), the growth and subsequent involution of several transient compartments (intermediate zone [IZ] and subplate [SP]), and the expansion and folding of the cortical plate (Vasung et al., 2016, 2019a). While these volumetric analyses provide valuable insights into brain growth, they are insensitive to many cellular events that occur during this critical period in development.

Diffusion tensor imaging (DTI) offers complementary information to structural imaging by probing tissue characteristics such as cellularity, water content, and tissue coherence. Due to the challenges related to subject motion, the early studies on fetal DTI consisted of ex vivo analysis. Huang and Vasung (2014) showed that DTI could demonstrate the various layers of the cerebral wall in fetuses between 13 and 21 weeks of GA and that deterministic tractography of major white matter bundles was possible even at this early GA. Song et al. (2015), utilized ex vivo high angular resolution diffusion (HARDI) tractography to characterize the longitudinal developmental trajectories and lateral asymmetries in major white matter pathways in fetuses, newborns, and infants; the focus of this study was on whole-tract analysis along a broad lifespan ( $>3$ years) and did not consider anatomical changes that occur in fetal life, such as the growth and involution of the transient white matter zones and the heterochronous onset of myelination. The study by Xu et al. (2014) correlated the tissue coherence pattern observed in HARDI tractography to immunohistochemical stains for vimentin (marker of cytoskeletal proteins of radial glial fibers), CD31 (vessel wall marker), and SMI320 (panaxonal neurofilament marker), and established that the radial glial fibers are the main determinant of anisotropy in the white matter of second trimester fetuses, thereby linking the process of neuronal migration and the regression of this glial scaffolding to changes in DTI parameters.

The continuous and unpredictable patterns of fetal motion in vivo pose a difficult technical problem for the acquisition and reconstruction of fetal brain DTI, requiring the utilization of several strategies. Jiang et al. (2009) proposed the first method to address this by combining data from axial slices using a slice-to-volume registration approach and data interpolation. Oubel, Koob, Studholme, Dietemann, and Rousseau (2012) used separate groupwise registration of the $b=0$ images and the diffusion sensitized images to T2-weighted fetal brain images. Fogtmann et al. (2014) built on this approach and coupled the registration process with a model of the imaging point spread function that utilized slices acquired in various planes. More recently, Marami et al. (2017) proposed a framework that incorporated two important concepts: first, interslice motion estimation through temporal slice registration coupled with a model of fetal head motion; and second, rejection of motion corrupted data in conjunction with a weighted linear least squares estimation to minimize the effect of signal loss. Our group has utilized the framework proposed by Marami et al. to perform in vivo tractography in individual subjects and analyze maturational changes that occur in the third trimester (Jaimes et al., 2020). These analyses included wholetract (tract-average) metrics and were constrained to a developmental period where myelination is known to drive the changes in diffusivity and anisotropy. The physiologic changes in the transient fetal compartments that occur in the second and early third trimester and how these shape local diffusivity properties of the traversing white matter tracts remain unexplored.

Population averaged diffusion atlases can be utilized to characterize complex microstructural changes with less bias than data from individual subjects (Yeh et al., 2018). This is particularly important when characterizing data sets with relatively low SNR, such as fetal DTI. The first reported application of atlas-based techniques to characterize microstructural fetal brain development were based on ex vivo data (Yu et al., 2016). Khan and colleagues utilized data from individual fetuses processed with the framework introduced by Marami et al. (2017) to create the first atlas of fetal DTI using in vivo data; this atlas depicts microstructural changes in high anatomic detail across a broad prenatal age range (Khan et al., 2019). The purpose of this study is to utilize these atlases of fetal brain DTI to investigate tract-based changes with high spatiotemporal resolution. We hypothesized that the atlas-based tractography would be able to separately demonstrate the effects of the involution of the transient fetal zones and the subsequent onset of myelination. Furthermore, we expected to be able to investigate the local (within-tract) developmental changes that drive tract-averaged microstructural changes.

\section{2 | MATERIALS AND METHODS}

\subsection{Study population}

This was a HIPAA compliant study approved by the Institutional Review Board of Boston Children's Hospital; all methods were performed in accordance with the relevant guidelines and regulations. Participants were prospectively recruited and informed consent was obtained prior to the MRI. Criteria for inclusion were: (a) normal pregnancy between 20 and 40 weeks of gestational age (GA), (b) maternal age between 18 and 45 years, and (c) lack of significant prior medical history in the mother. Exclusion criteria were: (a) maternal contraindication to MRI, (b) suspected or confirmed congenital infection in the fetus, (c) brain or other organ abnormalities in the fetus (known or identified on the fetal MRI), (d) chromosomal aberrations, and (e) multiple-gestation pregnancy. 


\subsection{Image acquisition}

Subjects were imaged on 3 Tesla (T) MRI scanners (Skyra or Prisma, Siemens Medical Solutions, Erlangen, Germany) using spine and 18-channel body coils. The structural imaging protocol consistent of multiple T2-weighted Half-Fourier-Acquired Single-shot Turbo spin Echo (HASTE) scans in orthogonal planes, with the following acquisition parameters: time to repetition $(T R)=1,400-2,000 \mathrm{~ms}$, Time to echo $(T E)=100-120 \mathrm{~ms}, 2$ or 4 slice interleaved acquisitions, 0.9$1.1 \mathrm{~mm}$ in-plane resolution, $2-3 \mathrm{~mm}$ slice thickness without gap, matrix size $=256 \times 204,256 \times 256$, or $320 \times 320$ with. The DTI protocol consisted of three to eight scans, also along orthogonal planes with respect to the fetal head. In each scan, 1 or $2 b=0 \mathrm{~s} /$ $\mathrm{mm}^{2}$ images, and 12 diffusion-sensitized images at $b=500 \mathrm{~s} / \mathrm{mm}^{2}$ were acquired. Acquisition parameters were: $T R=3,000-4,000 \mathrm{~ms}$, $\mathrm{TE}=60 \mathrm{~ms}$, in-plane resolution $=2 \mathrm{~mm}$, slice thickness $=2-4 \mathrm{~mm}$.

\section{3 | Image processing}

Structural images for individual subjects were processed according to a previously validated slice-to-volume reconstruction (SVR) algorithm that uses 2D low-resolution HASTE images to generate 3D super resolution and intensity normalized volumetric image of the fetal brain (Kainz et al., 2015). The fetal DTI data were processed as described by Marami et al. (2016), with a robust motion-tracked (MT)-SVR approach; this reconstruction method discards motion corrupted diffusion sensitized images, registers the noncorrupted data to a common space, and leverages the redundancy obtained through serial/ repeated acquisitions to allow for a robust estimation of the diffusion tensor, despite partial rejection of data (Marami et al., 2017).

\subsection{DTI atlas construction}

A spatiotemporal atlas of fetal brain DTI was created using the data from individual subjects. The framework utilized was described by Khan et al. (2019), and consists of a tensor-to-tensor diffeomorphic deformable registration integrated with kernel regression in age (Khan et al., 2019). To build the atlas, we utilized images from 67 high quality examinations with optimal MT-SVR diffusion reconstructions (no significant blurring or distortions). These were used to generate 16 templates, each corresponding to a specific week of GA. The number and sex of subjects included for each gestational week template is described in Table 1.

\section{5 | Tractography processing and tract segmentation}

Each individual gestational week diffusion tensor template was processed using Diffusion Toolkit (Version 0.6.4.1, Athinoula A. Martinos Center for Biomedical Imaging, Massachusetts General
TABLE 1 Subjects of DTI atlas

\begin{tabular}{|c|c|c|}
\hline Gestational week & Number of subjects & Sex (males) \\
\hline 23 & 8 & $5^{a}$ \\
\hline 24 & 9 & $6^{\mathrm{a}}$ \\
\hline 25 & 8 & 5 \\
\hline 26 & 11 & 4 \\
\hline 27 & 11 & 4 \\
\hline 28 & 9 & 7 \\
\hline 29 & 11 & 8 \\
\hline 30 & 10 & 7 \\
\hline 31 & 10 & 9 \\
\hline 32 & 9 & 7 \\
\hline 33 & 9 & $6^{\mathrm{a}}$ \\
\hline 34 & 7 & $4^{a}$ \\
\hline 35 & 5 & 5 \\
\hline 36 & 5 & 4 \\
\hline 37 & 5 & 4 \\
\hline 38 & 5 & 4 \\
\hline
\end{tabular}

Abbreviation: DTI, diffusion tensor imaging.

${ }^{\text {aAt }}$ least one subject with unknown sex at time of scan.

Hospital, Boston, MA; Wang, Benner, Sorensen, \& Wedeen, 2007) with a deterministic fiber assignment by a continuous tracking (FACT) algorithm. A $35^{\circ}$ angle threshold and automatic FA thresholding were used to perform tractography by user-defined regions of interest (ROIs) (Wang et al., 2007). A research fellow with medical training drew inclusion and exclusion ROIs TrackVis (Trackvis 0.6.1 Athinoula A. Martinos Center for Biomedical Imaging, Massachusetts General Hospital; Wang et al., 2007) to delineate the forceps major, forceps minor, bilateral corticospinal tracts (CST), bilateral inferior frontooccipital fasciculus (IFOF), bilateral inferior longitudinal fasciculus (ILF), and bilateral uncinate fasciculus (UF) as previously described by de Macedo Rodrigues et al. (2015) and Catani and Thiebaut de Schotten (2008). This set of adjusted labels was shown to result in high-quality fetal tractography by Jaimes et al. (2020).

We performed whole-tract analysis by extracting the mean $F A$ and $A D C$ for each individual week template and tract. To evaluate local variation in diffusion metrics we performed a sub analysis of three tracts: the CST, forceps major, and IFOF. These tracts were selected because they represent projection, commissural, and association fibers, respectively, and have been reported to change rapidly with GA prenatally (Jaimes et al., 2020). These tracts were segmented into various components using ITK-SNAP (Yushkevich, Gao, \& Gerig, 2016). The CST was divided in an inferior segment extending from the medulla inferiorly to the midbrain superiorly (terminating at the level of the red nucleus and cerebral peduncle), a mid-segment extending from the lower aspect of the internal capsule to the inferior edge of the corpus callosum, and a superior segment extending from the corpus callosum to the cerebral cortex. 
The IFOF was subdivided into a rostral segment extending from the frontal cortex to the genu of the internal capsule, a mid-segment extending from the genu of the internal capsule to the periatrial white matter at the level of the splenium of the corpus callosum, and a caudal segment extending from here to the occipital cortex. The forceps major was divided into a central segment and two (right/left) hemispheric segments that extended from the temporo-occipital fissure to the cortex. The output of the ROI based tractography and the segmental tract analysis was reviewed and revised as needed by a board certified pediatric neuroradiologist with experience in fetal neuroimaging.

\subsection{Statistical analysis}

A linear regression analysis was used to evaluate gestational age as predictor of FA and MD. Laterality (left or right) was included as a covariate for CST, IFOF, ILF, and UF. The relationship between
FA/MD and gestational age was modeled using linear or quadratic terms, as determined by visual inspection of scatterplots and overall fit of the model as measured by adjusted R (Bystron et al., 2008). For quadratic models, results were reported for $\leq 30$ gestational weeks (point estimate centered at 27 weeks) and for $>30$ gestational weeks (point estimate centered at 34.5 weeks). This division was determined a priori as it represents the time at which the transient fetal white matter zones start to involute, and then was confirmed by inspection of the experimental data. Level of significance was set at an $\alpha$ value of .05 for all tests. Stata 15/EC (StataCorp, College Station, TX) was used for all tests and graphs.

\section{3 | RESULTS}

All white matter tracts were successfully delineated in the GA-specific diffusion templates. Figure 1 illustrates robust outcome of tractography across the GAs studied.

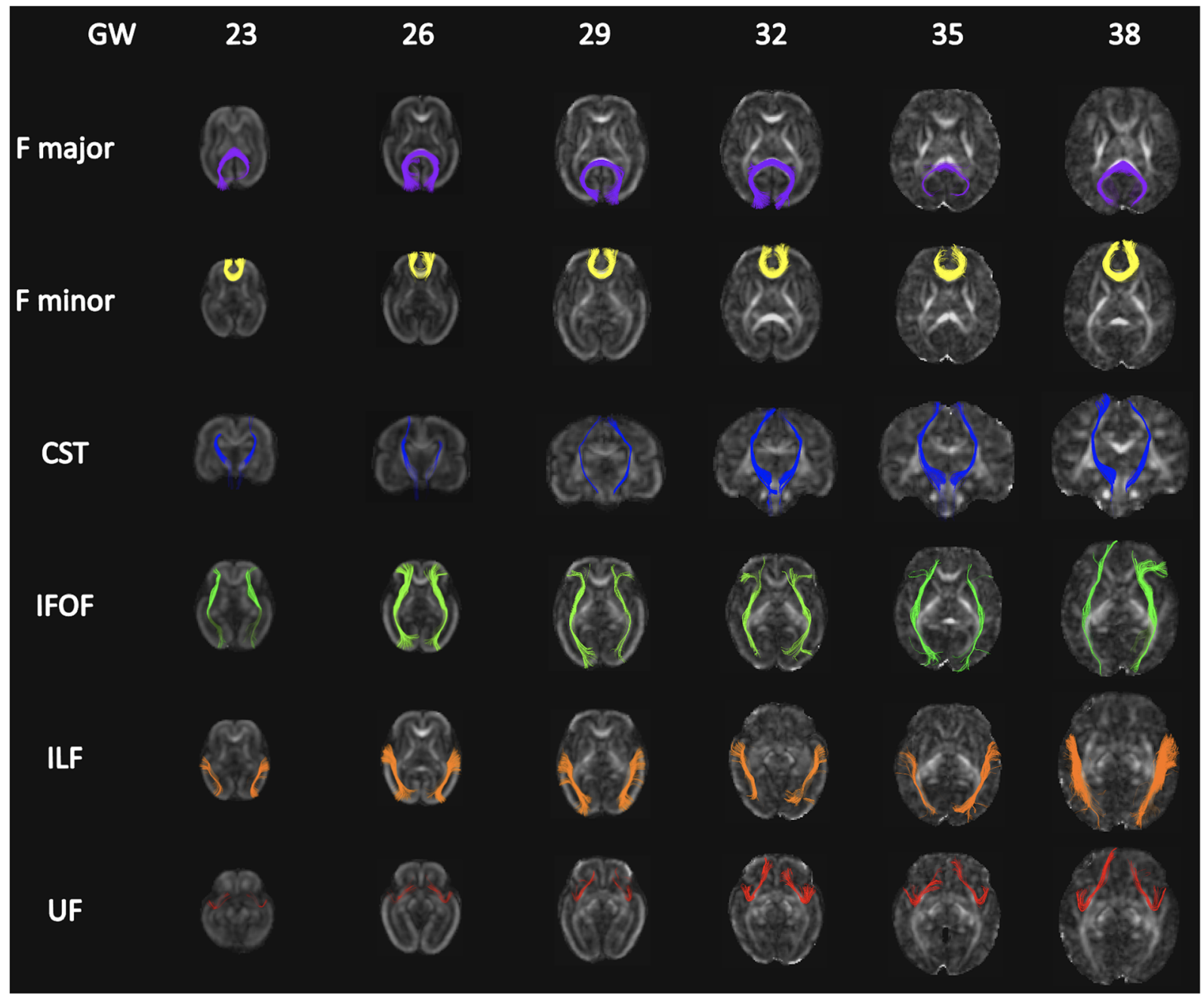

FIGURE 1 Longitudinal tractography of the fetal DTI templates. Deterministic tractography on selected GA templates; tracts are superimposed on the corresponding FA map. The outlined tracts include forceps major ( $F$ major), forceps minor ( $F$ minor), corticospinal tract (CST), inferior fronto-occipital fasciculus (IFOF), inferior longitudinal fasciculus (ILF), and uncinate fasciculus (UF). GW, gestational weeks 


\section{1 | Whole-tract analysis}

\subsection{1 | Commissural tracts}

Forceps major and forceps minor tracts were delineated for all gestational ages (Table 2); there was no premature termination of tractography, with both ends of each tract extending to the cortex. In the forceps major, FA significantly increased across all GAs studied $(p<.001)$. In the forceps minor, FA significantly decreased with GA at $\leq 30$ weeks $(p=.003$ ), while in GA $>30$ weeks FA change was not significant ( $p=0.532$; Figure 2a,b).

In the forceps major MD significantly decreased with GA at $>30$ weeks $(p<.001)$, but change was not significant at GA at $\leq 30$ weeks $(p=.134)$. In the forceps minor, MD increased significantly with GA at $\leq 30$ weeks $(p<.001$ ), and significantly decreased with GA at $>30$ weeks $(p=0.006)$ (Figure $2 c, d)$.

\subsection{2 | Projection tracts}

Left and right CST were delineated for all gestational ages (Table 2). In templates for low GA ( $<27$ weeks), the tracts did not reach the cortical plate and the tractography terminated the intermediate zone, before reaching the SP. In templates for the rest of GAs, tractography reached the cortical plate. At $\leq 30$ weeks there was no significant change in FA with increasing GA $(p=.620)$. FA significantly increased with $G A$ at $>30$ weeks $(p<.001)$. There was no significant difference in FA $(p=.886)$ between right and left tracts accounting for GA (Figure 3a).

MD did not significantly increase with GA at $\leq 30$ weeks ( $p=.109$ ), but significantly decreased with $\mathrm{GA}>30$ weeks $(p<.001)$. Similarly, there was no significant difference in MD $(p=.960)$ between right and left tracts accounting for GA (Figure 3b).

\subsection{3 | Association tracts}

Left and right IFOF, ILF, and UF were delineated for all gestational ages (Table 2). In the IFOF, FA significantly decreased with GA at $\leq 30$ weeks ( $p=.011$ ), and significantly increased with GA at $>30$ weeks $(p<.001)$. There was no significant difference in FA between right and left IFOF accounting for GA ( $p=.062)$. In the ILF, FA significantly decreased with GA at $\leq 30$ weeks $(p<.001)$, and significantly increased with GA at $>30$ weeks $(p<.001)$. There was no significant difference in FA between right and left ILF accounting for GA ( $p=$.189). In the UF, FA was not significantly associated with GA at $\leq 30$ weeks $(p=.807)$, but significantly

TAB LE 2 Whole-tract analysis of rate of microstructure change

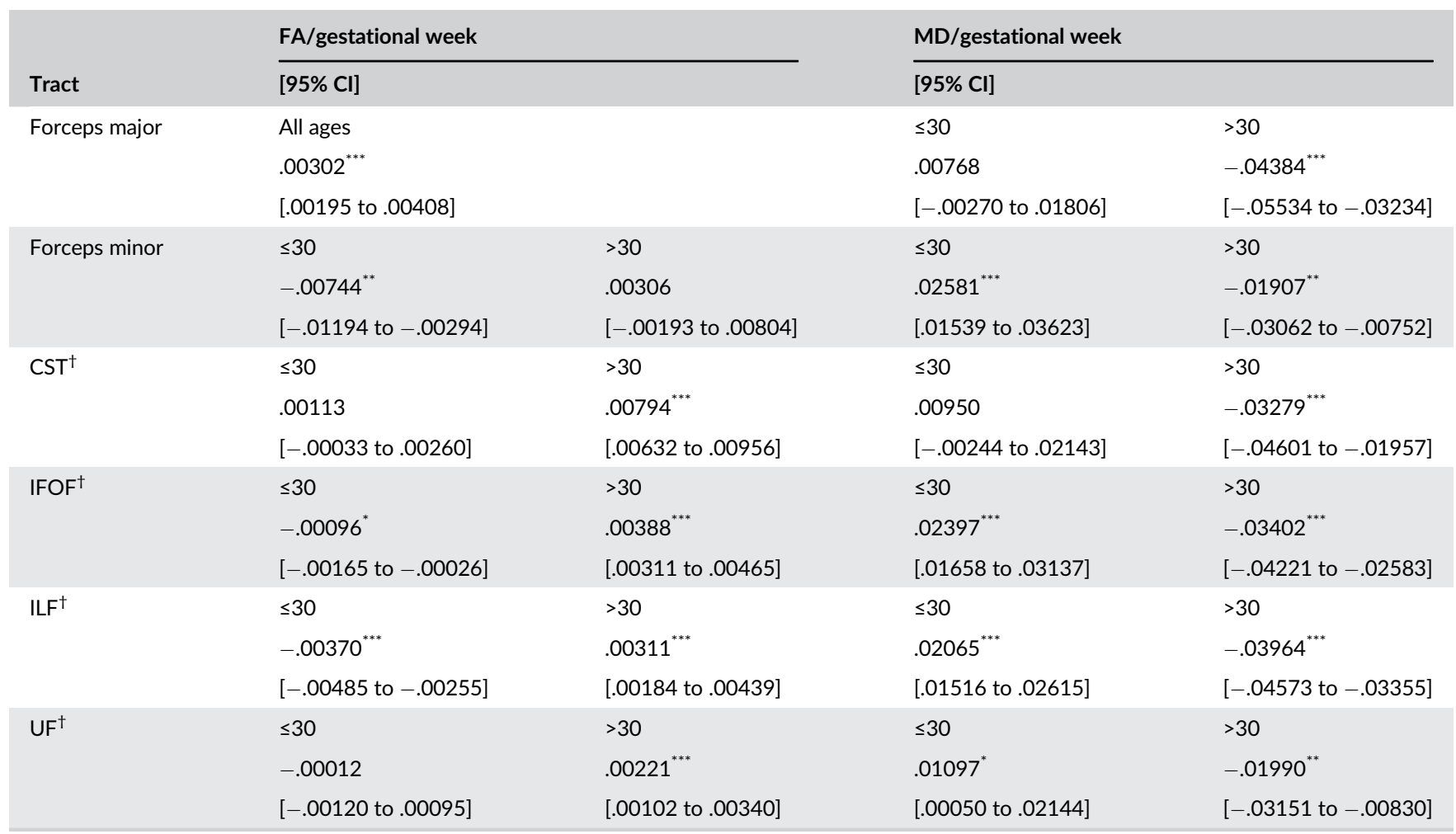

Note: Point estimates for $\leq 30$ weeks centered at 27 weeks, and for $>30$ weeks centered at 34.5 weeks.

Abbreviations: CST, corticospinal tract; FA, fractional anisotropy, IFOF, inferior fronto-occipital fasciculus; ILF, inferior longitudinal fasciculus; MD, mean diffusivity; UF, uncinate fasciculus.

${ }^{*} p<.050 .{ }^{* *} p<.010 .{ }^{* * *} p<.001 .{ }^{\dagger} \mathrm{FA}$ and MD of left and right tracts not significantly different $(p>.050)$. 

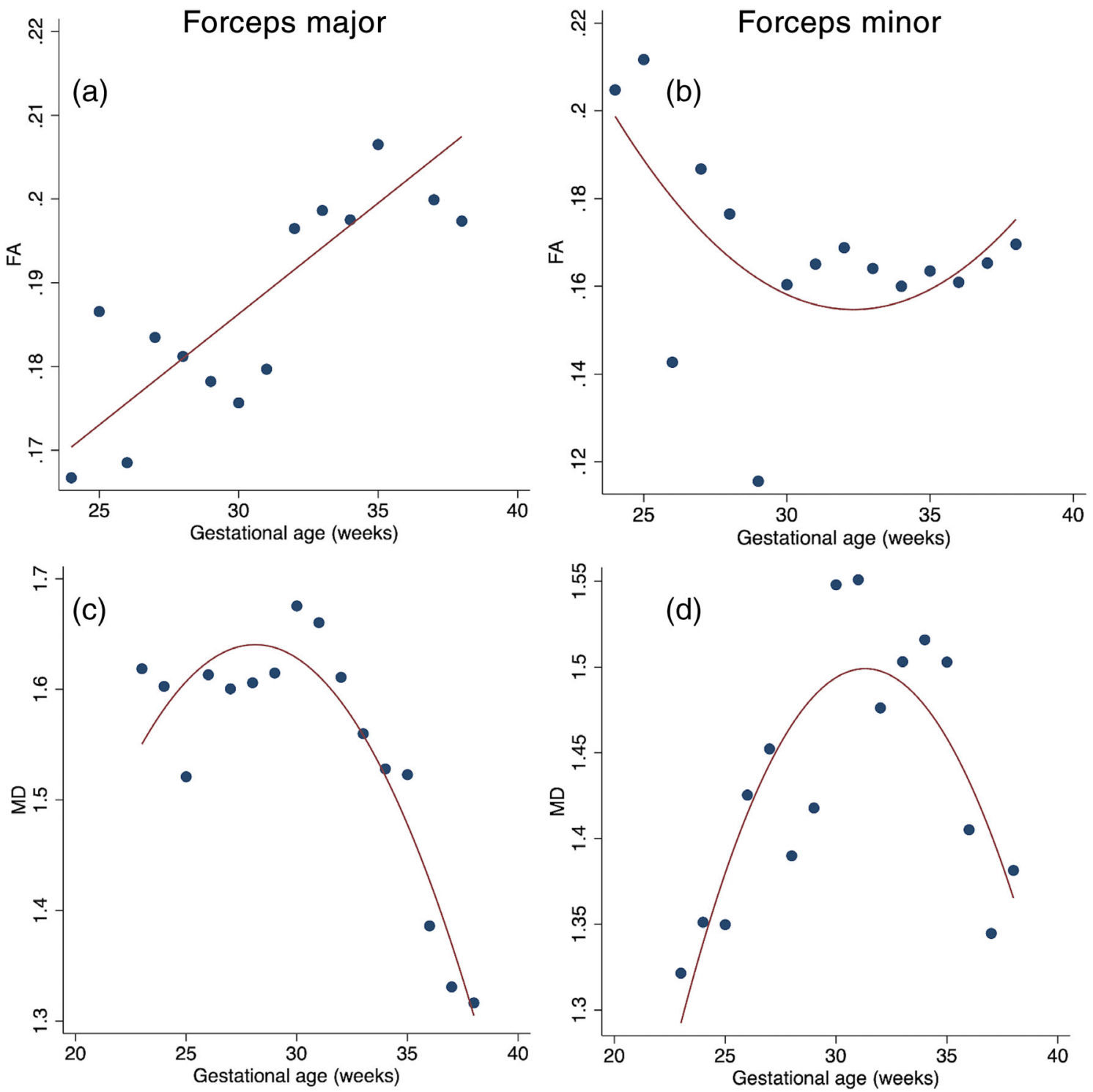

FIGURE 2 Whole-tract analysis of commissural tracts. GA-related changes in FA and MD in the forceps major (a,c) and forceps minor (b,d). Regression model using linear terms (a) and quadratic terms (b-d) are shown. Rate of change and statistical significance are shown in Table 2

increased with GA at $>30$ weeks $(p<.001)$. As before, there was no significant difference in FA between right and left UF accounting for GA $(p=.990)$ (Figure 4a-c).

MD significantly increased in the IFOF with GA at $\leq 30$ weeks $(p<.001)$, and significantly decreased with GA at $>30$ weeks $(p<.001)$. There was no significant difference in MD between right and left IFOF accounting for GA ( $p=.081)$. For the ILF, MD significantly increased with GA at $\leq 30$ weeks $(p<.001)$, and significantly decreased with GA at $>30(p<.001)$. Similarly, there was no significant difference in MD between right and left ILF accounting for GA ( $p=.887)$. For the UF, MD significantly increased with GA at $>30$ weeks $(p=.041)$, and significantly decreased with GA at $\leq 30$ weeks ( $p=.003$ ). Again, there was no significant difference in MD between right and left ILF accounting for $\mathrm{GA}(p=.845$; Figure $4 \mathrm{~d}-\mathrm{f})$.

\subsection{Local changes (within-tract analysis)}

\subsection{1 | Commissural tracts}

Forceps major segments (central and peripheral) were analyzed for all GA (Table 3). FA increase in the central segment was not statistically significant $(p=.106)$. In the peripheral segments, FA significantly increased with GA at $\leq 30$ weeks $(p<.001)$, but was not significantly associated with GA at $>30$ weeks $(p=.877)$.

$M D$ for the central segment did not change significantly with $G A$ at $\leq 30$ weeks $(p=.215$ ), while it significantly decreased with GA $>30$ weeks $(p=.002)$. For the peripheral segment, MD significantly increased with $\mathrm{GA} \leq 30$ weeks ( $p=.003$ ), and significantly decreased with $\mathrm{GA}$ at $>30$ weeks $(p<.001$; Figure 5$)$. 

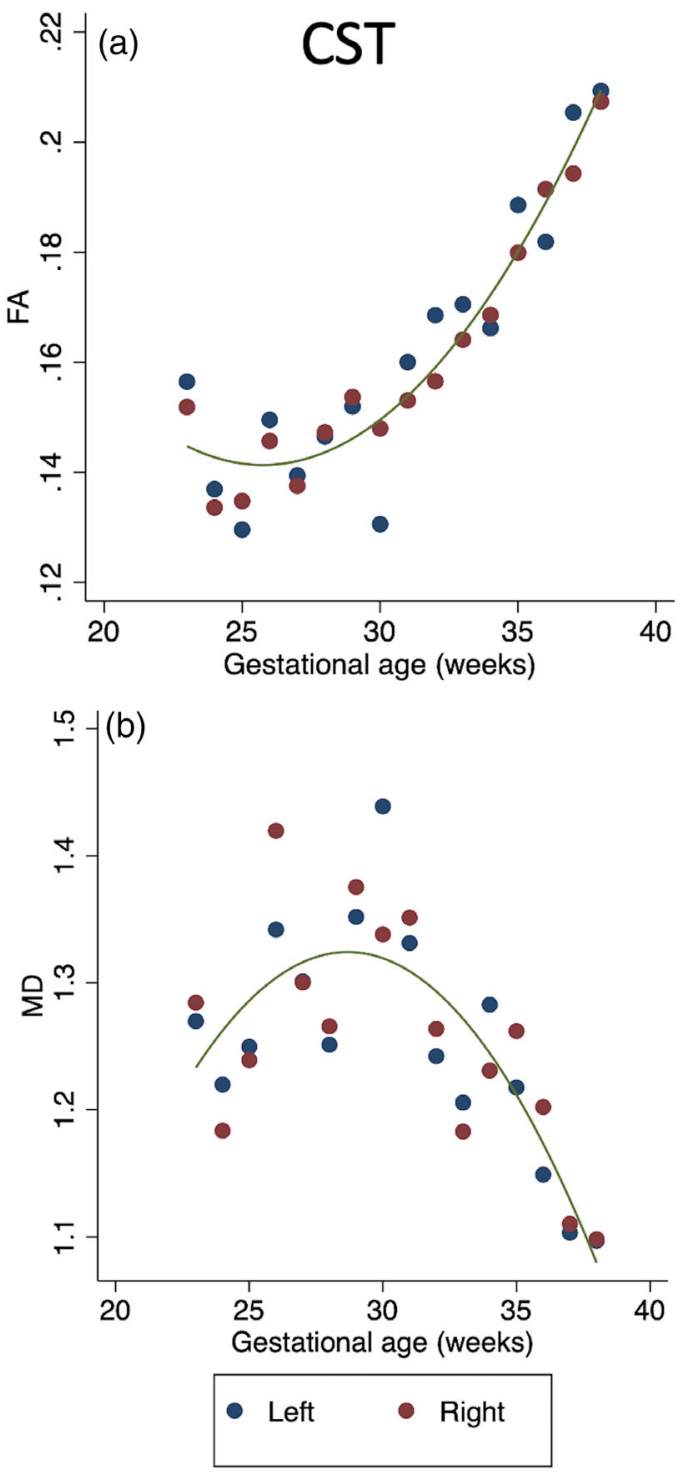

FIGURE 3 Whole-tract analysis of projection tracts. GA-related changes in FA and MD in the CST. Regression models are shown, rate of change and significance is shown in Table 2. Laterality was included in the regression model, but was not statistically significant $(p>.05)$

\subsection{2 | Projection tracts}

CST segments (superior, middle, and inferior) were analyzed for all GA (Table 3). FA in the superior segment significantly decreased with GA at $\leq 30$ weeks $(p<.001)$, and significantly increased with GA at $>30$ weeks $(p<.001)$. In the middle segment, FA significantly increased with all GA $(p<.001)$. In the inferior segment, FA significantly increased with GA at $\leq 30$ ( $p=.017)$, but was not significantly associated with $\mathrm{GA}$ at $>30$ weeks $(p=.494)$.

In the superior segment MD was not significantly associated with $\mathrm{GA}$ at $\leq 30$ weeks $(p=.936$ ), but significantly decreased at $>30$ weeks ( $p=.012$ ). Similarly, MD in the middle segment was not significantly associated with GA at $\leq 30$ weeks ( $p=.447$ ), but significantly decreased at $>30$ weeks $(p<.001)$. In the inferior segment, MD was not significantly associated with GA ( $p=.298$; Figure 6).

\subsection{3 | Association tracts}

Results for the individual segments (rostral, middle, and caudal) of the IFOF are summarized in Table 3. FA in the rostral segment significantly decreased with GA $\leq 30$ weeks $(p<.001$ ), but was not significantly associated with GA at $>30$ weeks $(p=.631)$. In the middle segment, FA was not significantly associated with GA at $\leq 30$ weeks $(p=.680)$, but significantly increased with GA at $>30$ weeks ( $p=.001$ ). In the caudal segment, FA significantly decreased with GA at $\leq 30 \quad(p<.001)$, and significantly increased with GA at $>30$ weeks $(p<.001)$.

Comparatively, the MD in the rostral segment significantly increased with GA at $\leq 30$ weeks $(p<.001)$, and significantly decreased with GA at $>30$ weeks $(p=.005)$. Similarly, in the middle segment MD significantly increased with GA at $\leq 30$ weeks ( $p$ <.001), and significantly decreased with GA at >30 weeks $(p<.001)$. In the caudal segment, MD also significantly increased with $G A \leq 30$ weeks $(p<.001)$, and significantly decreased with GA $>30$ weeks $(p<.001)$ (Figure 7$)$.

\section{DISCUSSION}

The advent of motion robust fetal DTI permitted in vivo characterization of brain microstructural development (Jaimes et al., 2020). The construction of spatiotemporal atlases of fetal brain DTI have further enabled analysis of many developmental changes at early GAs despite the small size of the structures and the relatively low SNR of the individual acquisitions. In this study, we utilized an atlas-based approach to evaluate tract-specific microstructural changes and their relationship to other important cytoarchitectonic processes. Our atlas-based diffusion tractography shows a series of tract-specific, age-related changes in FA and MD. While the changes in the later part of gestation ( $>30$ weeks) conform to expected postnatal trajectories depicting an increase in FA and a decrease in $M D$, these trends are unapparent or appear reversed in several major white matter tracts earlier in gestation (2229 weeks). These age-related changes in diffusion properties are consistent with the observations reported by Wilson et al. (2021). This divergence indicates that at earlier developmental stages different biologic processes drive diffusivity and anisotropy in the parenchyma and consequently along the traversing white matter tracts. In addition, our segment-based analysis of selected commissural, projection, and association tracts demonstrates considerable within-tract heterogeneity in maturation, with distinct local developmental trajectories.

Two anatomically overlapping yet biologically distinct and asynchronous processes take place in the brain in the second half 

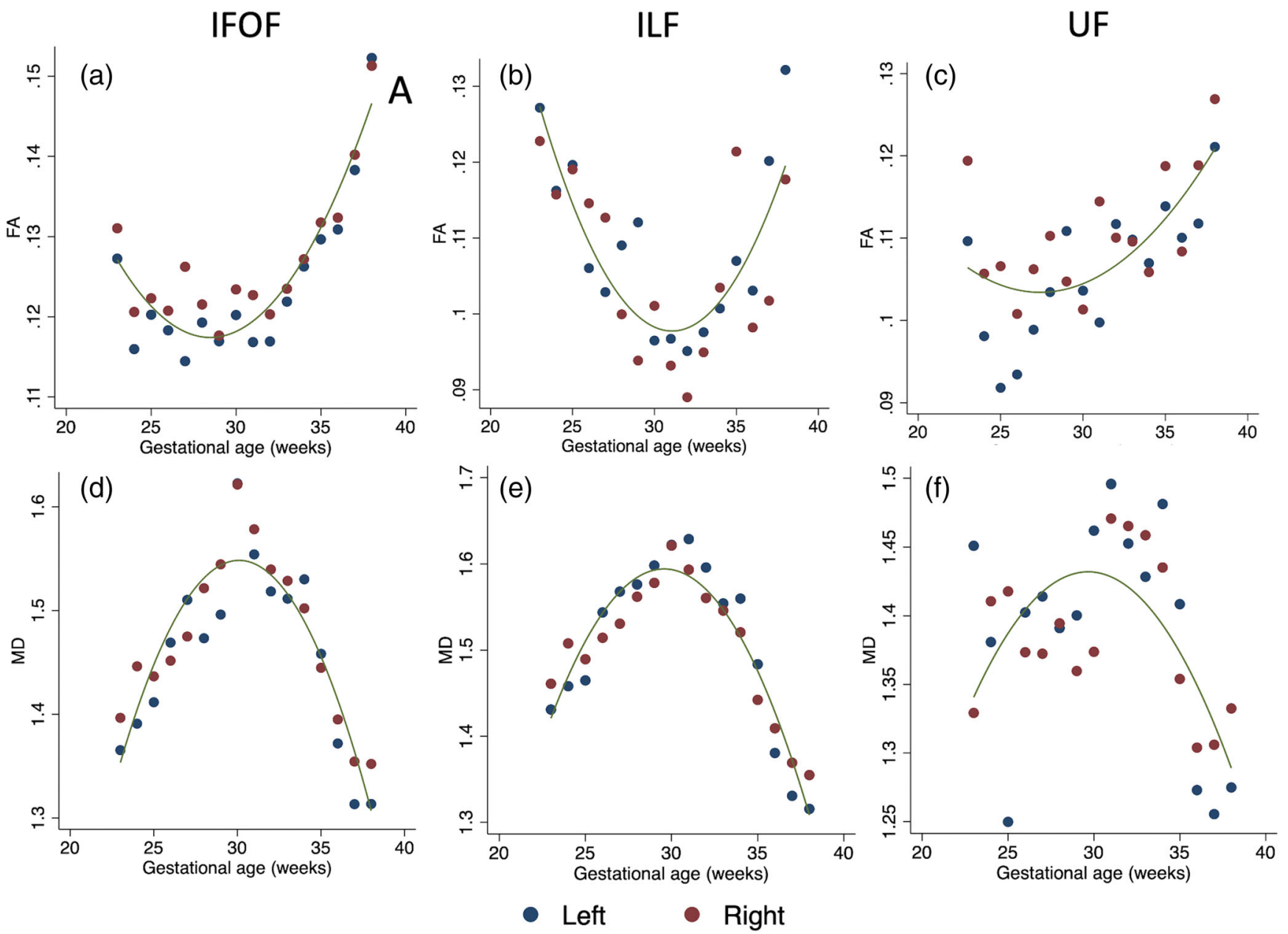

FIGURE 4 Whole-tract analysis of association tracts. GA-related changes in FA and MD in the inferior fronto-occipital fasciculus (IFOF) (a,d), inferior longitudinal fasciculus (ILF) (b,e), and uncinate fasciculus (UF) (c,f). Regression models are shown, rate of change and significance are shown in Table 2. Laterality was included in the regression model but was not statistically significant $(p>.05)$ for any tract

of gestation: (a) the evolution of the transient zones of the fetal telencephalon and (b) the onset and progression of myelination. Early in the second trimester, the telencephalon is organized in concentric layers, most of which are visible on MRI. The outermost layer or cortical plate (CP) contains postmigratory neurons and shows high FA and low MD early in gestation; as the CP matures, dendritic arborization and synapse formation mediate an increase in intercellular space that leads to loss of FA and higher diffusivity (McKinstry et al., 2002). Beneath the CP, there is a water rich and relatively acellular layer characterized by high MD and very low FA, known as the SP (Widjaja et al., 2010; Xu et al., 2014). The SP increases in thickness and volume during the second and early third trimester, with a reported peak at approximately 30 weeks of GA when it occupies up to $45 \%$ of the volume of the telencephalon (Huang \& Vasung, 2014). The IZ is the deep layer subjacent to the SP, which contains migrating neurons and organized axonal bundles that result in moderately low MD and high FA. Overtime, the loss of the radial glial scaffolding and the dispersion of the migrating neurons results in a loss of FA and increase in MD (Widjaja et al., 2010; Xu et al., 2014). After 30 weeks of GA, the SP and IZ undergo reorganization and become difficult to differentiate on MRI; this process has been described by Kostovic et al. as the "resolution of the subplate" (Kostovic \& Rakic, 1990), which ultimately results in consolidation of these two layers to form the "mature" fetal cerebral white matter (Vasung et al., 2019b). Independent of these changes, myelination begins at approximately 25 weeks of $\mathrm{GA}$, with the earliest detectable histologic findings in the posterior limb of the internal capsules, posterior globus pallidus, and ventral lateral thalamus (Jaimes et al., 2020; Kinney, Brody, Kloman, \& Gilles, 1988). From there on, myelination progresses along white matter tracts in caudal-to-rostral, central-to-peripheral, and anterior-toposterior direction (Abe, Takagi, Yamamoto, Okuhata, \& Kato, 2004; Counsell et al., 2002). It is well-known that myelination results in increase in FA and decreased MD (Neil et al., 1998). 
TAB LE 3 Within tract analysis of rate of microstructure change

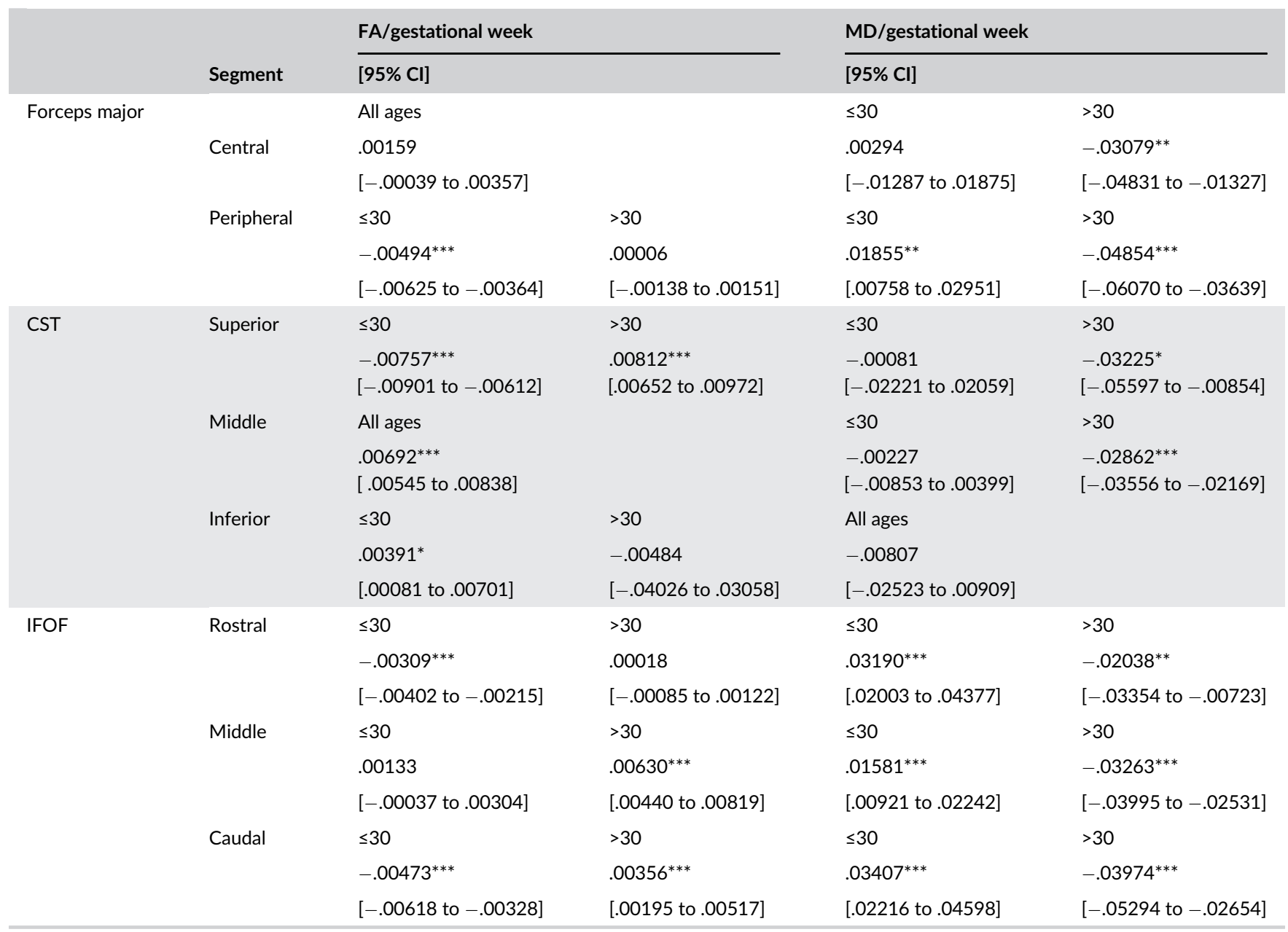

Note: Point estimates for $\leq 30$ weeks centered at 27 weeks, and for $>30$ weeks centered at 34.5 weeks.

Abbreviations: CST, corticospinal tract; FA, fractional anisotropy; IFOF, inferior fronto-occipital fasciculus; MD, mean diffusivity. ${ }^{*} p<.050 .{ }^{* *} p<.010 .{ }^{* * *} p<.001$.

The nonlinear GA- and tract-specific trends observed in our tractography analysis reflect the varying influence of these processes on the diffusivity characteristics of the fetal brain. The distinct GA-related increase in FA and decrease in MD that is seen in all tracts after 30 weeks of GA is consistent with the anatomic progression of myelination (Jaimes et al., 2020). Even though some premyelination and myelination changes are known to occur prior to this age, the consolidation of this trend appears to coincide chronologically with the "resolution stage" of the fetal SP and IZ as well as more widespread and robust myelination on histology (Brody et al., 1987; Kinney et al., 1988). At GA less than 30 weeks, the microstructural changes of the transient fetal zones play a dominant role. For most tracts (forceps minor, ILF, IFOF, and UF), there was a significant decrease in FA and increase in MD with GA in this time period. These changes correspond with the layer-specific diffusion and volumetric changes described above (see paragraph above). Specifically, the age-related decrease in FA in the cortex and $\mathrm{IZ}$ and the expansion of the SP (with its low FA and high MD), contribute to these observations. Given that the long white matter bundles traverse these layers, the large effects of the layer-specific microstructural properties on whole-tract estimates is expected (Figure S1).

Tractography of the CST deserves special mention. In fetuses $<27$ weeks, the CST tracking terminates at the interface between the IZ and the SP, without appreciable fiber tracking along the SP or extension to the primary motor cortex. Review of the raw data, with lax deterministic tractography parameters (no exclusion ROIs), shows crossing fibers headed toward the lateral parietal and frontal regions. This is probably secondary to fiber tracking along the radial glial scaffolding that predominates in the second trimester; the former, in conjunction with the inherent limitation of the diffusion tensor model to resolve crossing fibers, results in the incomplete delineation of the tract (Xu et al., 2014; Figure S2). The missing segment of the tract corresponds to the SP and CP voxels; given the 


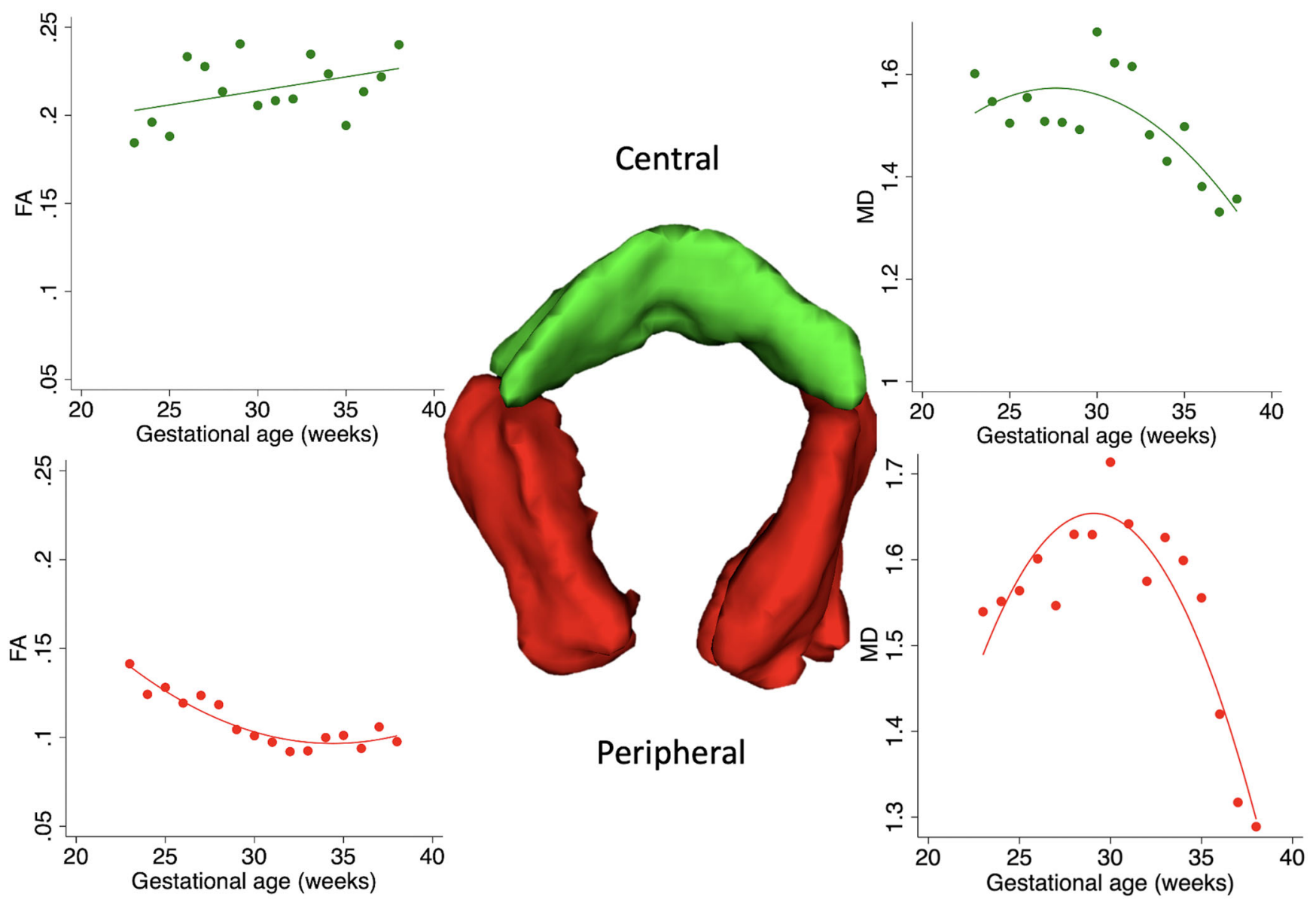

FIGURE 5 Within-tract analysis of forceps major. Microstructural changes in the central (green) and peripheral (red) segments of the forceps major. The central segment shows gestational age (GA)-related increase in FA whereas the peripheral segment shows a decrease in FA. The MD of both segments shows a similar pattern, with a peak around 30 weeks of gestational age (GA). Rate of change and significance are shown in Table 3

large relative volume of the SP in this developmental stage and the dominant diffusion features of this layer, we believe that this contributes to the lack of significant change in FA or MD before 30 weeks of GA.

We also observed substantial within tract heterogeneity in microstructural development of the CST, forceps major, and IFOF. The presence of distinct developmental trajectories for segments within a single white matter tract suggests that tract averaged $M D$ and FA are insufficient to capture the complex developmental changes of fetal brain. The advantage of more precise spatial analyses has been documented postnatally. Colby et al. (2012) showed that within-tracts analysis outlined microstructural differences in children with fetal alcohol spectrum disorders that were not apparent on the tract-averaged analyses. Our data, albeit limited to selected white matter tracts, are similar. For instance, most of the peripheral/telencephalic components of tracts (CST: superior segment; IFOF: rostral and caudal segments; forceps major: peripheral segments), showed an inflection point or nadir in FA at 29-32 weeks of GA. These findings were not apparent on tract-averaged analysis of the CST or forceps major as they were probably averaged with other components of the tracts. The central components of the tracts (CST: mid segment; IFOF: mid segment; forceps major: central segment) showed different trends. In all of these, the FA showed a continued linear increase with age, an effect that is likely dominated by density of axonal packing and myelination, as these segments do not traverse the telencephalic layers. The forceps major does show a peak around 30 weeks, that is likely driven by the other effects of axonal organization. Finally, the caudal segment of the CST shows the fastest rate of increase in FA of all CST segments at $\leq 30$ weeks of $G A$, which is consistent with the early myelination of the brainstem which precedes supratentorial myelination; subsequently, the rate of change decreases as myelination of these segment of the CST is largely complete by the mid third trimester. The set of ROIs chosen for this analysis are based solely on anatomy; however, future work will be 
(a)
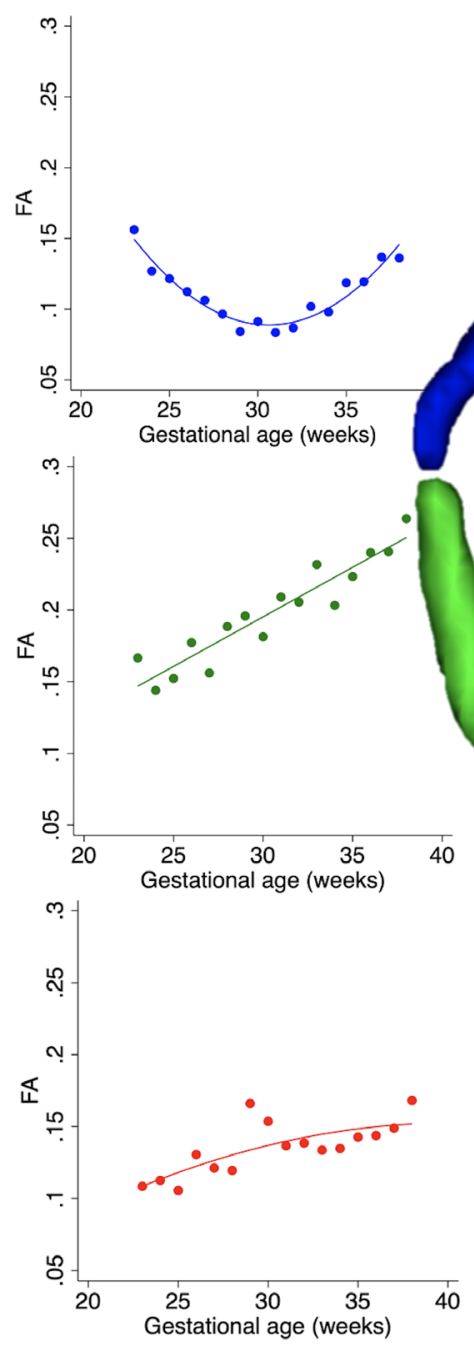

\section{Superior}
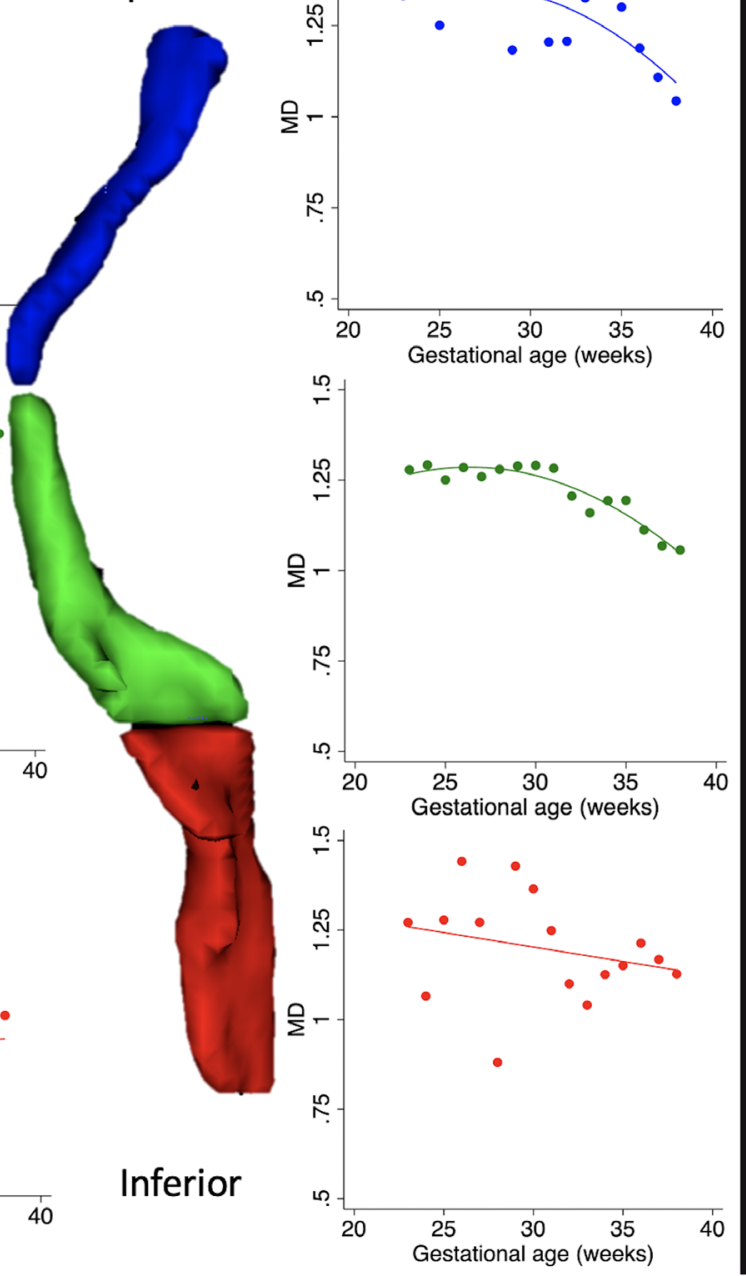

\section{Inferior}

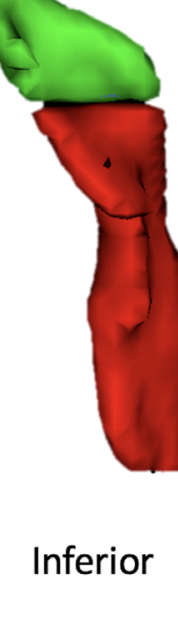

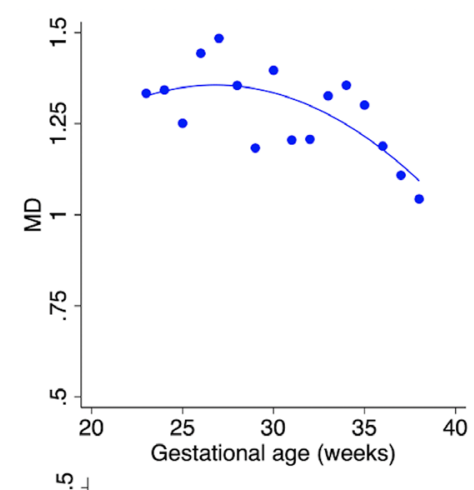

(b)

(2)

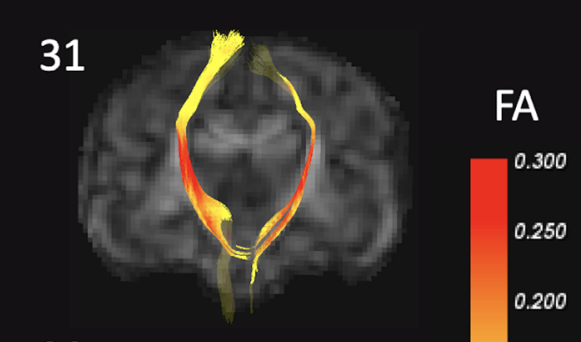

38

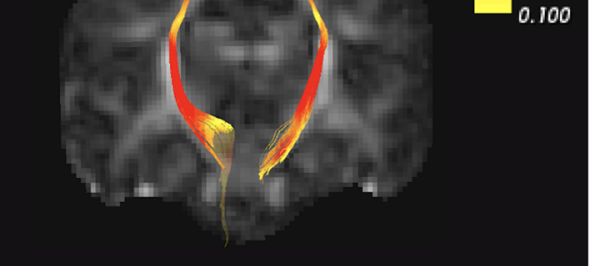

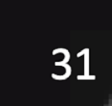
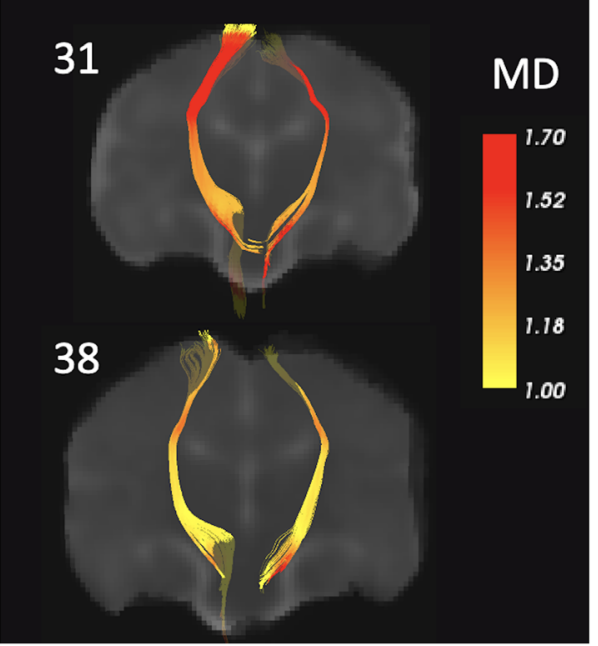

FIGURE 6 Within-tract analysis of CST. Heterogeneity in development of the CST. (a) Microstructural changes in the superior (blue), mid (green), and inferior (red) segments of the CST. The superior segment shows nadir in FA at approximately 30 weeks of gestational age (GA). The mid segment shows linear GA-related increase in FA and decrease in MD. The inferior segment shows an increase in FA early in the second trimester with lower rate of change later in gestation. Rates of change and significance are shown in Table 3. (b) Heat maps of FA and MD at 31 and 38 weeks of GA show within tract heterogeneity in progression of microstructural changes

focused on developing robust tools for along tract analysis that incorporate growth and change in geometry of individual white matter tracts. Improvement in these tools will improve our ability to correlate these findings with patterns of gene expression, early functional specialization, and selective vulnerability to injury.

This study has several limitations. Our analysis is based on a single-tensor model of the diffusion signal. This approach is sufficient to characterize tissue coherence along major white matter tracts; however, it imposes limitations related to crossing fibers which, as our data show, can impact the ability to delineate the anatomy of fetal white matter tracts and impact the reliability of the microstructural analysis. Future work utilizing other models of diffusion signal processing, including diffusion compartment imaging, are expected to improve our ability to resolve these structures (Scherrer et al., 2016). Additionally, our analysis of within tract changes are based on anatomic parcellations of the white matter; although these convincingly demonstrate the heterogeneity of the white matter maturation, they do not perform direct spatial correspondence or enable group wise comparisons. Development of novel methods that allow for such analyses, analogous to those existing for postnatal pipelines (Colby et al., 2012), will significantly improve our ability to study brain maturation in utero. 

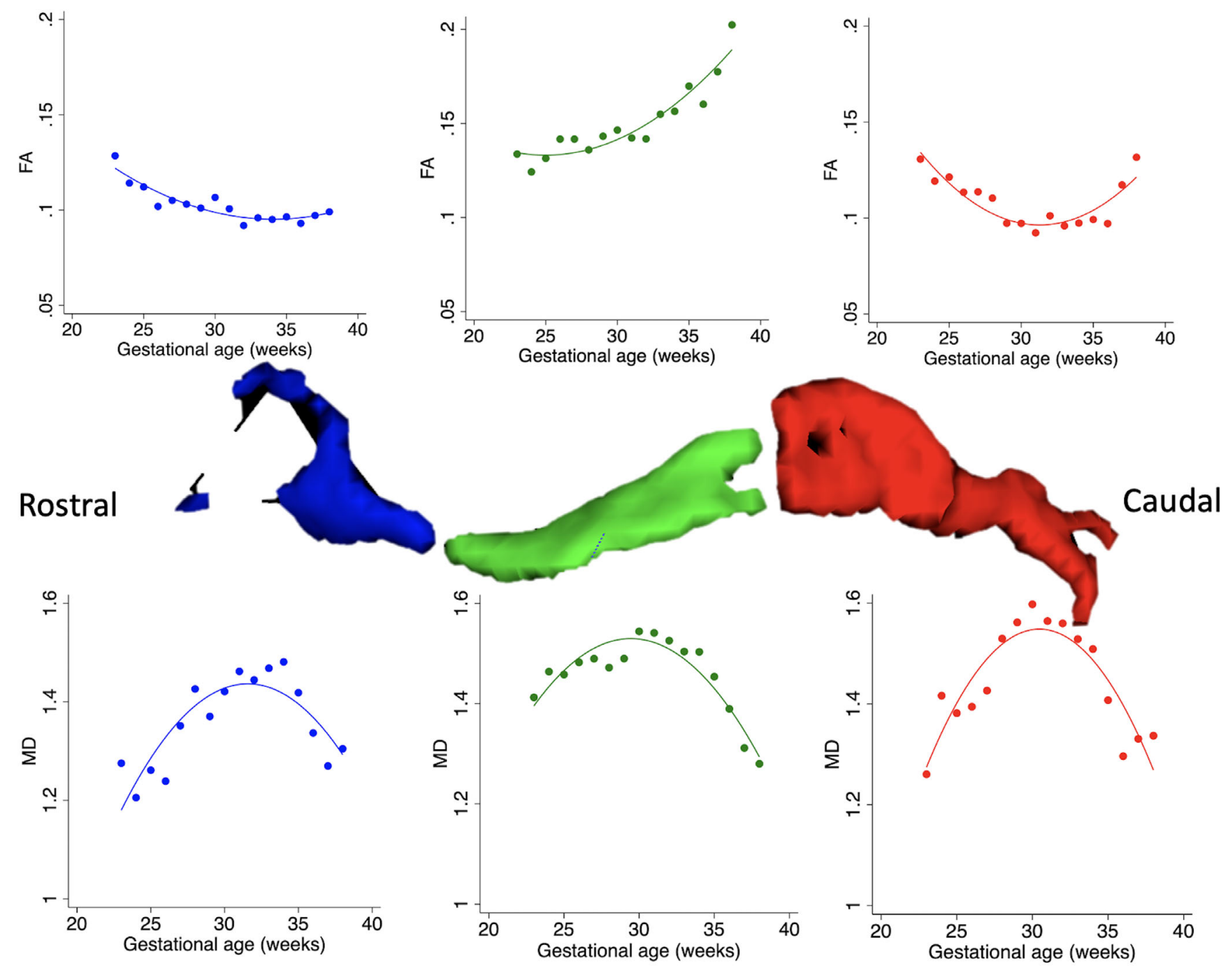

FIG URE 7 Within-tract analysis of IFOF. Microstructural changes in the rostral (blue), mid (green), and caudal (red) segments of the IFOF. The rostral and caudal segments show a decrease in FA at $\leq 30$ weeks of GA probably as a result of expansion of the subplate. After $>30$ weeks of GA, the caudal segment shows GA-related increase in FA that exceeds that of the rostral segment, representing the expected progression of myelination. The mid segment shows a continuous GA-related increase in FA as it is not influenced by changes in transient telencephalic zones. For all segments, the MD shows central peak, although the trajectories are slightly different. Rates of change and significance are shown in Table 3

\section{5 | CONCLUSION}

Diffusion-atlas based tractography shows the white matter cytoarchitectonics drive diffusivity and anisotropy in the second and early third trimester and that myelination becomes a dominant factor in the mid to late third trimester. Our analysis also showed significant within-tract heterogeneity in development, highlighting the need to develop tools that permit robust along-tract analyses.

\section{ACKNOWLEDGMENT}

Research reported in this publication was supported in part by the National Institute of Biomedical Imaging and Bioengineering, the National Institute of Neurological Disorders and Stroke, and the National Institute of Dental and Craniofacial Research of the National Institutes of Health (NIH) under award numbers R01EB031849,
R01NS106030, R01EB018988, R01EB013248, and R03DE022109; in part by the Office of the Director of the NIH under Award Number S10OD0250111; and in part by a Technological Innovations in Neuroscience Award from the McKnight Foundation. The content of this publication is solely the responsibility of the authors and does not necessarily represent the official views of the $\mathrm{NIH}$, the McKnight Foundation, or the American Roentgen Ray Society.

\section{CONFLICT OF INTEREST}

The authors declare no potential conflict of interest.

\section{DATA AVAILABILITY STATEMENT}

The data that support the findings of this study are openly available at http://crl.med.harvard.edu/research/fetal_brain_atlas/ as part of Fetal Diffusion Tensor Imaging (DTI) Atlas. 


\section{ORCID}

Fedel Machado-Rivas (D) https://orcid.org/0000-0003-3095-414X

Onur Afacan (D) https://orcid.org/0000-0003-2112-3205

Simon K. Warfield (iD) https://orcid.org/0000-0002-7659-3880

Ali Gholipour (D) https://orcid.org/0000-0001-7699-4564

Camilo Jaimes (D) https://orcid.org/0000-0002-7169-4244

\section{REFERENCES}

Abe, S., Takagi, K., Yamamoto, T., Okuhata, Y., \& Kato, T. (2004). Semiquantitative assessment of myelination using magnetic resonance imaging in normal fetal brains. Prenatal Diagnosis, 24, 352-357.

Andescavage, N. N., du Plessis, A., McCarter, R., Serag, A., Evangelou, I., Vezina, G., ... Limperopoulos, C. (2016). Complex trajectories of brain development in the healthy human fetus. Cerebral Cortex, 27(11), 5274-5283. https://doi.org/10.1093/cercor/bhw306

Brody, B. A., Kinney, H. C., Kloman, A. S., \& Gilles, F. H. (1987). Sequence of central nervous system myelination in human infancy. I. An autopsy study of myelination. Journal of Neuropathology and Experimental Neurology, 46, 283-301.

Bystron, I., Blakemore, C., \& Rakic, P. (2008). Development of the human cerebral cortex: Boulder Committee revisited. Nature Reviews. Neuroscience, 9, 110-122.

Catani, M., \& Thiebaut de Schotten, M. (2008). A diffusion tensor imaging tractography atlas for virtual in vivo dissections. Cortex, 44, 11051132.

Colby, J. B., Soderberg, L., Lebel, C., Dinov, I. D., Thompson, P. M., \& Sowell, E. R. (2012). Along-tract statistics allow for enhanced tractography analysis. Neurolmage, 59(4), 3227-3242. https://doi.org/ 10.1016/j.neuroimage.2011.11.004

Counsell, S. J., Maalouf, E. F., Fletcher, A. M., Duggan, P., Battin, M., Lewis, H. J., ... Rutherford, M. A. (2002). MR imaging assessment of myelination in the very preterm brain. American journal of neuroradiology, 23(5), 872-881.

de Macedo Rodrigues, K., Ben-Avi, E., Sliva, D. D., Choe, M.-S., Drottar, M., Wang, R., ... Zöllei, L. (2015). A FreeSurfer-compliant consistent manual segmentation of infant brains spanning the 0-2 year age range. Frontiers in Human Neuroscience, 9. https://doi.org/10.3389/fnhum. 2015.00021

Fogtmann, M., Seshamani, S., Kroenke, C., Xi, C., Chapman, T., Wilm, J., ... Studholme, C. (2014). A Unified approach to diffusion direction sensitive slice registration and 3-d dti reconstruction from moving fetal brain anatomy. IEEE Transactions on Medical Imaging, 33(2), 272-289. https://doi.org/10.1109/tmi.2013.2284014

Huang, H., \& Vasung, L. (2014). Gaining insight of fetal brain development with diffusion MRI and histology. International Journal of Developmental Neuroscience, 32, 11-22.

Jaimes, C., Rofeberg, V., Stopp, C., Ortinau, C. M., Gholipour, A., Friedman, K. G. ... Rollins, C. K. (2020). Association of isolated congenital heart disease with fetal brain maturation. American Journal of Neuroradiology, 41(8), 1525-1531. https://doi.org/10.3174/ajnr. a6635

Jaimes, C., Machado-Rivas, F., Afacan, O., Khan, S., Marami, B., Ortinau, C. M., ... Gholipour, A. (2020). In vivo characterization of emerging white matter microstructure in the fetal brain in the third trimester. Human Brain Mapping, 41(12), 3177-3185. https://doi. org/10.1002/hbm.25006

Jiang, S., Xue, H., Counsell, S., Anjari, M., Allsop, J., Rutherford, M., ... Hajnal, J. V. (2009). Diffusion tensor imaging (DTI) of the brain in moving subjects: Application to in-utero fetal and ex-utero studies. Magnetic Resonance in Medicine, 62(3), 645-655. https://doi.org/10.1002/ mrm.22032

Kainz, B., Steinberger, M., Wein, W., Kuklisova-Murgasova, M., Malamateniou, C., Keraudren, K., ... Rueckert, D. (2015). Fast volume reconstruction from motion corrupted stacks of 2D slices. IEEE Transactions on Medical Imaging, 34(9), 1901-1913.

Khan, S., Vasung, L., Marami, B., Rollins, C. K., Afacan, O., Ortinau, C. M., ... Gholipour, A. (2019). Fetal brain growth portrayed by a spatiotemporal diffusion tensor MRI atlas computed from in utero images. Neurolmage, 185, 593-608. https://doi.org/10.1016/j.neuroimage. 2018.08.030

Kinney, H. C., Betty, B. A., Kloman, A. S., \& Gilles, F. H. (1988). Sequence of central nervous system myelination in human infancy. ii. patterns of myelination in autopsied infants. Journal of Neuropathology and Experimental Neurology, 47(3), 217-234. https://doi.org/10.1097/ 00005072-198805000-00003

Kostovic, I., \& Rakic, P. (1990). Developmental history of the transient subplate zone in the visual and somatosensory cortex of the macaque monkey and human brain. Journal of Comparative Neurology, 297, 441-470.

Marami, B., Scherrer, B., Afacan, O., Erem, B., Warfield, S. K., \& Gholipour, A. (2016). Motion-robust diffusion-weighted brain mri reconstruction through slice-level registration-based motion tracking. IEEE Transactions on Medical Imaging, 35(10), 2258-2269. https://doi. org/10.1109/tmi.2016.2555244

Marami, B., Mohseni Salehi, S. S., Afacan, O., Scherrer, B., Rollins, C. K., Yang, E., ... Gholipour, A. (2017). Temporal slice registration and robust diffusion-tensor reconstruction for improved fetal brain structural connectivity analysis. Neurolmage, 156, 475-488. https://doi.org/10. 1016/j.neuroimage.2017.04.033

McKinstry, R. C., Mathur, A., Miller, J. H., Ozcan, A., Snyder, A. Z., Schefft, G. L., ... Neil, J. J. (2002). Radial organization of developing preterm human cerebral cortex revealed by non-invasive water diffusion anisotropy MRI. Cerebral Cortex, 12(12), 1237-1243. https://doi.org/ 10.1093/cercor/12.12.1237

Neil, J. J., Shiran, S. I., McKinstry, R. C., Schefft, G. L., Snyder, A. Z., Almli, C. R., ... Conturo, T. E. (1998). Normal brain in human newborns: apparent diffusion coefficient and diffusion anisotropy measured by using diffusion tensor MR imaging. Radiology, 209(1), 57-66. https:// doi.org/10.1148/radiology.209.1.9769812

Oubel, E., Koob, M., Studholme, C., Dietemann, J.-L., \& Rousseau, F. (2012). Reconstruction of scattered data in fetal diffusion MRI. Medical Image Analysis, 16, 28-37.

Scherrer, B., Schwartzman, A., Taquet, M., Sahin, M., Prabhu, S. P., \& Warfield, S. K. (2016). Characterizing brain tissue by assessment of the distribution of anisotropic microstructural environments in diffusioncompartment imaging (DIAMOND). Magnetic Resonance in Medicine, 76(3), 963-977. https://doi.org/10.1002/mrm.25912

Song, J. W., Mitchell, P. D., Kolasinski, J., Ellen, G. P., Galaburda, A. M., \& Takahashi, E. (2015). Asymmetry of white matter pathways in developing human brains. Cerebral Cortex, 25(9), 2883-2893. https://doi.org/ 10.1093/cercor/bhu084

Vasung, L., Lepage, C., Radoš, M., Pletikos, M., Goldman, J. S., Richiardi, J. ... Kostovic, I. (2016). Quantitative and qualitative analysis of transient fetal compartments during prenatal human brain development. Frontiers in Neuroanatomy, 10. https://doi.org/10.3389/fnana.2016. 00011

Vasung, L., Charvet, C. J., Shiohama, T., Gagoski, B., Levman, J., \& Takahashi, E. (2019). Ex vivo fetal brain MRI: Recent advances, challenges, and future directions. Neurolmage, 195, 23-37. https://doi. org/10.1016/j.neuroimage.2019.03.034

Vasung, L., Rollins, C. K, Yun, H. J., Velasco-Annis, C., Zhang, J., Wagstyl, K. ... Gholipour, A. (2020). Quantitative in vivo MRI assessment of structural asymmetries and sexual dimorphism of transient fetal compartments in the human brain. Cerebral Cortex, 30(3), 1752-1767. https:// doi.org/10.1093/cercor/bhz200

Wang, R., Benner, T., Sorensen, A. G., \& Wedeen, V. J. (2007). Diffusion toolkit: A software package for diffusion imaging data processing and tractography. Proceedings of the International Society for Magnetic 
Resonance in Medicine, 15, 3720. http://trackvis.org/faq/2007 ISMRM_diffusion_toolkit.pdf

Widjaja, E., Geibprasert, S., Mahmoodabadi, S. Z., Blaser, S., Brown, N. E., \& Shannon, P. (2010). Alteration of human fetal subplate layer and intermediate zone during normal development on $\mathrm{mr}$ and diffusion tensor imaging. American Journal of Neuroradiology, 31(6), 1091-1099. https://doi.org/10.3174/ajnr.a1985

Wilson, S., Pietsch, M., Cordero-Grande, L., Price, A. N., Hutter, J., Xiao, J. ... O'Muircheartaigh, J. (2021). Development of human white matter pathways in utero over the second and third trimester. Proceedings of the National Academy of Sciences, 118(20), e2023598118. https://doi. org/10.1073/pnas.2023598118

Xu, G., Takahashi, E., Folkerth, R. D., Haynes, R. L., Volpe, J. J., Grant, P. E., \& Kinney, H. C. (2014). Radial coherence of diffusion tractography in the cerebral white matter of the human fetus: neuroanatomic insights. Cerebral Cortex, 24(3), 579-592. https://doi.org/10.1093/cercor/ bhs330

Yeh, F. C., Panesar, S., Fernandes, D., Meola, A., Yoshino, M., FernandezMiranda, J. C., ... Verstynen, T. (2018). Population-averaged atlas of the macroscale human structural connectome and its network topology. Neurolmage, 178, 57-68. https://doi.org/10.1016/j.neuroimage. 2018.05.027

Yu, Q., Ouyang, A., Chalak, L., Jeon, T., Chia, J., Mishra, V. S., ... Huang, H. (2016). Structural development of human fetal and preterm brain cortical plate based on population-averaged templates. Cerebral Cortex, 26(11), 4381-4391. https://doi.org/10.1093/cercor/bhv201

Yushkevich, P. A., Yang, G., \& Gerig, G. (2016). ITK-SNAP: An interactive tool for semi-automatic segmentation of multi-modality biomedical images. Annual International Conference of the IEEE Engineering in Medicine and Biology Society. IEEE Engineering in Medicine and Biology Society, Orlando, FL. 3342-3345. https://doi.org/10.1109/EMBC. 2016.7591443

\section{SUPPORTING INFORMATION}

Additional supporting information may be found in the online version of the article at the publisher's website.

How to cite this article: Machado-Rivas, F., Afacan, O., Khan, S., Marami, B., Velasco-Annis, C., Lidov, H., Warfield, S. K., Gholipour, A., \& Jaimes, C. (2021). Spatiotemporal changes in diffusivity and anisotropy in fetal brain tractography. Human Brain Mapping, 42(17), 5771-5784. https://doi.org/10.1002/ hbm.25653 